\title{
Centella asiatica in cosmetology
}

\author{
Wiesława Bylka, Paulina Znajdek-Awiżeń, Elżbieta Studzińska-Sroka, Małgorzata Brzezińska
}

Department of Pharmacognosy, Poznan University of Medical Sciences, Poland

Head: Prof. Irena Matławska

Postep Derm Alergol 2013; XXX, 1: 46-49

DOI: 10.5114/pdia.2013.33378

\begin{abstract}
Centella asiatica known as Gotu Kola is a medicinal plant that has been used in folk medicine for hundreds of years as well as in scientifically oriented medicine. The active compounds include pentacyclic triterpenes, mainly asiaticoside, madecassoside, asiatic and madecassic acids. Centella asiatica is effective in improving treatment of small wounds, hypertrophic wounds as well as burns, psoriasis and scleroderma. The mechanism of action involves promoting fibroblast proliferation and increasing the synthesis of collagen and intracellular fibronectin content and also improvement of the tensile strength of newly formed skin as well as inhibiting the inflammatory phase of hypertrophic scars and keloids. Research results indicate that it can be used in the treatment of photoaging skin, cellulite and striae.
\end{abstract}

Key words: Centella asiatica, triterpenes, wound healing, cosmetic preparation.

\section{Introduction}

Centella asiatica (L) Urban., synonym: Hydrocotyle asiatica L. (Eng. Indian Pennywort, Fr. Hydrocotyle asiatique, Ger. Asiatischer Wassernabel), also known by common names as: Gotu kola or Tiger Grass, belongs to the Apiaceae family. It grows in Asia, mainly in India, Pakistan, Madagascar, equatorial Africa, central America and in the tropical region of Oceania [1]. The C. asiatica herb was already used as a "panacea" three thousand years ago in China, India, Africa, the Philippines, Sri Lanka and Madagascar. In the $19^{\text {th }}$ century, $C$. asiatica and C. asiatica extract were included in the Indian Pharmacopoeia. Due to the medicinal properties of the plant it was gradually incorporated into other Pharmacopoeias: British Herbal Pharmacopoeia, Chinese Pharmacopoeia, Dutch Pharmacopoeia, German Pharmacopoeia, Homöopathisches Arzneibuch, Martindale European Pharmacopoeia, Pharmacopee française, La Farmacopea Italiana X, European Pharmacopoeia VI and into Polish Pharmacopoeia IX edition [1-3]. According to European and Polish Pharmacopoeias, Centella (Centellae asiaticae herba) consists of dried, fragmented aerial parts of Centella asiatica (L Urban.), contains not less than 6.0 per cent of total triterpenoid derivatives expressed as asiaticoside [2, 3].

In traditional Asian medicine, the herb of $C$. asiatica has been used for hundreds of years, especially in dermatolo- gical conditions, to improve small wounds, scratches, burns, hypertrophic wounds healing, and as an anti-inflammatory agent, particularly in eczema. It is also recommended as an antipyretic, diuretic, rheumatic, antibacterial, antiviral drug, in the treatment of vein insufficiency and for improving cognition, relieving anxiety and as an anti-cancer agent $[1,4,5]$. Formerly, C. asiatica was also used in epilepsy, hysteria, leprosy, and in minor itching and insect bites [5].

This review presents the results of activity of $C$. asiatica in facilitating the healing of wounds, compounds responsible for activity and mechanism of action, also contraindications and potential side effects. So far studies in the use of this plant as an ingredient in cosmetic preparations applied on photoaging skin, in cellulite and striae have also been presented.

\section{Chemical composition}

The most important constituents isolated from C. asiatica were triterpenoid saponins known as centelloids. Saponins may account for $1 \%$ to $8 \%$ of all C. asiatica constituents [6]. The variable quantity mainly depends on the origin of the plant and can be established using the HPLC-UV method [7].

Centellosides are primarily ursane- and oleanane-type pentacyclic triterpenoid saponins. The most important compounds, due to their pharmacological activity, are asiati-

Address for correspondence: Prof. Wiesława Bylka, Department of Pharmacognosy, Poznan University of Medical Sciences, 4 Swiecickiego Str., 60-781 Poznan, Poland, phone: +48 6185467 09, e-mail: wieslawabylka@tlen.pl

Received: 13.08.2012, accepted: 24.10.2012. 
coside, madecassoside, asiatic acid and madecassic acid. Other centellosides occurring in C. asiatica include triterpenic acids, for example: brahmic acid, madasiatic acid, terminolic acid, centellic acid as well as their glycosides: brahminoside, madasiaticoside and centelloside [6]. Centella also contains other components, including volatile oils (0.1\%), flavonoids, tannins, phytosterols, amino acids and sugars $[1,8]$.

\section{Preparations}

Pharmacological, biochemical and clinical studies were related to undefined aqueous or alcohol extracts and defined extracts. Pharmacological research included the following extracts: titrated extract of Centella asiatica (TECA), total triterpenoid fraction of Centella asiatica (TTFCA), both containing asiatic acid (30\%), madecassic acid (30\%), asiaticosides (40\%) and total triterpenic fraction (TTF) which comprises asiatic acid and madecassic acid (60\%) as well as asiaticosides (40\%) [1]. However, information coming from licensed medicinal products says that all of the above mentioned TECA, TTFCA, TTF as well as CATTF and ETCA are different acronyms to designate the same extract, commercially known as Madecassol ${ }^{\circledR}$, Centellase ${ }^{\circledR}$ or Blastoestimulina ${ }^{\circledR}$, containing $40 \%$ of asiaticoside and a $60 \%$ mixture of asiatic and madecassic acids [1,9].

Clinical trials were performed using TECA and ETCA (Estratto Titolato di Centella asiatica) or commercially produced extracts: Centella asiatica total triterpenic fraction (CATTF) and TTFCA as well as isolated triterpenoid saponins, mainly asiaticoside, madecassoside and asiatic acid [1, 9].

The $C$. asiatica preparations used in conventional medicine are applied in an oral form (tablets and drops), topical medication (ointments and powder), in the form of injections (s.c., i.m.) and external or internal homeopathic preparations [1].

\section{Biological activity}

Centella asiatica is effective in treatment of wounds, even in infected wounds, as well as burns and postoperative hypertrophic scars [10-13]. Triterpene compounds: asiatic acid, madecassic acid, asiaticoside and madecassoside are the principal components of $C$. asiatica, responsible for wound healing. The action has been demonstrated both for the extracts as well as triterpene compounds in a large number of scientific reports involving in vitro and in vivo experiments [14, 15].

The terpenoids (TTF) cause a significant increase in the percentage of collagen and cell layer fibronectin. The most beneficial effects are the stimulation of scar maturation by the production of type I collagen, decrease in the inflammatory reaction and myofibroblast production [16].

According to Maquart et al., wounds treated with TECA and its separated components: asiatic acid, madecassic acid and asiaticoside increased total protein, collagen, and peptic hydroxyproline content. As a result, the increase of fibroblast proliferation by stimulation of cell migration from the surrounding tissues and/or the expression or activation of some growth factors in the wound was observed. The triterpenoid components were also able to stimulate glycosaminoglycan, especially hyaluronic acid synthesis [17].

The saponins from C. asiatica administered orally or subcutaneously to the rats, during 3 weeks caused the reduction of the scar granuloma, whereas the rupture, tensile strength and the collagen content in the scar tissue increased [1].

The ethanolic extract of the $C$. asiatica facilitated the wound-healing in both normal and dexamethasone suppressed wound healing. The study was done in Wistar albino rats using incision, excision and dead space wounds models. The extract increased the wound breaking strength in incision wound model, accelerated the epithelization and the rate of wound contraction. Granulation tissue and hydroxyproline content in a dead space wound also increased. The extract attenuated dexamethasone healing effect in all wound models [18].

Madecassol ${ }^{\circledR}$ was found to have a preventive effect on burn and postoperative hypertrophic scars [13].

Madecassoside was active in burn wound healing, through increasing antioxidative activity and enhancing collagen synthesis, and influencing angiogenesis. Oral administration of this compound at doses of $6 \mathrm{mg} / \mathrm{kg}$, $12 \mathrm{mg} / \mathrm{kg}$ and $24 \mathrm{mg} / \mathrm{kg}$ to mice facilitated wound closure in a time-dependent manner and complete wound closure took place on the $20^{\text {th }}$ day in the group receiving $24 \mathrm{mg} / \mathrm{kg}$ of madecassoside. The compound caused dermal proliferation of fibroblasts and increased the level of hydroxyproline (used as an indicator of collagen synthesis in burned skin) which resulted in enhanced epithelization. These results confirm a positive effect on fibroblast proliferation and collagen synthesis during burn wound repair [11].

Asiaticoside also stimulated angiogenesis so that the increase in tension and elasticity of blood vessels was observed [19].

Studies on rats have shown that this compound (0.2\%) applied topically increases the levels of enzymatic and non-enzymatic antioxidants in the newly created tissues [20].

Asiaticoside caused an increase in hydroxyproline and also in tensile strength, a rise in collagen content and better epithelization of punch/puncture wounds in guinea pigs or on delayed-type wounds of guinea pigs with experimentally induced diabetes [19].

The mechanism of action of asiaticoside is the induction of type I collagen synthesis in human dermal fibroblast cells by phosphorylation of both Smad 2 and Smad 3, also binding Smad 3 and Smad 4. Studies show that asiaticoside can induce type I collagen by activation of tumor growth factor $\beta$ (TGF- $\beta$ ) receptor I kinase - independent Smad pathway [21]. 
The facilitation of burn wound healing, observed after 20 days of local application of asiaticoside at a dose of $10 \mathrm{pg}, 1 \mathrm{ng}$, or $100 \mathrm{ng} /$ wound area on the backs of mice, took place through influence on the level of various cytokines produced in the place of the burn wound. The improvement in burn wound healing might be an outcome of angiogenesis promotion during wound healing in the injured area occurring as a result of the stimulation of vascular endothelial growth factor (VEGF) production [22].

Eventually, proliferation of epithelial cells and wound contraction occur. In contraction, the wound is made smaller by the activity of myofibroblasts [10-12].

Centella asiatica herb water extracts also shows the anti-psoriatic activity [23], exerts a beneficial effect in treating systemic scleroderma and focal scleroderma [24].

In cosmetology C. asiatica has been used as an effective anti-photoaging agent, mainly due to enhancement of type I collagen, which amount in skin decreases with age. The action was confirmed in a randomized, double blind clinical trial conducted among 20 female participants (45-60 years old) with photoaged skin to examine the impact of topically applied $0.1 \%$ madecassoside in conjunction with $5 \%$ vitamin C on their skin. Six-month treatment resulted in a significant improvement in firmness, elasticity and skin hydration, which was confirmed by appropriate biometrological tests. It is considered that the beneficial effect of $C$. asiatica on improving the condition of skin was due to madecassoside, a known inducer of collagen expression by activating the SMAD signaling pathway. In the previous study, the same investigators confirmed the beneficial effect of $5 \%$ vitamin C on photoaged skin, which resulted from stimulation of collagen synthesis in fibroblasts and control of matrix metalloproteinase enzymes responsible for degradation of collagen, while in photoaged skin, the level of vitamin C in tissues was significantly reduced. Thus, it follows that the mixture of vitamin $C$ and madecassoside is an attractive combination of two active compounds characterized by different mechanisms of activity, which exert an additive or synergistic effect "causing the remodeling of the superficial dermis" [25].

Centella asiatica is a common ingredient of cosmetics used in cellulite and striae. Cellulite, known as liposclerosis, is a non-inflammatory change within the subcutaneous adipose tissue caused by an increase in the volume of fat cells or by increased division of the connective tissue, which causes constriction of small blood vessels. The result is a disorder of metabolism in the subcutaneous adipose tissue, resulting in "distended" fat cells in this tissue, particularly around hips, buttocks, abdomen, thighs and arms. Cellulite is an ailment that affects many people, most frequently women, especially obese and leading stationary lifestyle, but may even be encountered in children.

Preparations that affect the adipose tissue, connective tissue and improve microcirculation are applied in the treatment of cellulite. They can be used topically, internally and transdermally. It is recommended to include medicines of plant origin in the fight against cellulite. Methylxanthines (caffeine, theobromine, theophylline) have a lipophilic effect, while the extract of $C$. asiatica normalizes the metabolism occurring in the cells of the connective tissue, shows anti-inflammatory and draining activity and regulates microcirculation.

The study confirmed the influence of triterpenes of C. asiatica on increasing the metabolism of lysine and proline, the amino acids building the collagen molecule. In addition, these compounds increased the synthesis of tropocollagen, and mucopolysaccharide in the connective tissues. The results obtained showed the impact of $C$. asiatica on improving nutrition of tissues and connective vascular stimulation [26].

The positive effects of the treatment of cellulite with the $C$. asiatica extract were confirmed by a few clinical studies that used different methodologies. These experiments, however, used non-standard criteria to evaluate the progress of the treatment and most of them lacked a control group. The histopathological study performed in a double-blind trial, involving 35 patients evaluated the size of adipocytes in the gluteofemoral region and in the deltoid region. Twenty people participating in the experiment were administered $60 \mathrm{mg}$ of the dry extract of $C$. asiatica orally once a day, for 90 days. Other participants of the experiment group took a placebo. The results showed that in the patients taking the Gotu Kola extract, the diameter of fat cells (or adipocytes) decreased in both studied regions of the body with a predominance of positive changes in the gluteofemoral region. In this study, a reduction in interadipocyte fibrosis was also observed [27].

In the experiment on a group of 60 people with cellulite, the influence of Madecassol ${ }^{\circledR}$ applied four times a day for 4 months was studied. The results clearly showed a beneficial effect of the Gotu Kola extract on inhibiting the progression of cellulite and a significant improvement in the skin condition in $85 \%$ of the experiment participants. The preparation was well tolerated [1].

Other experiments conducted on 65 patients with advanced cellulite showed that oral intake of the preparation Madecassol together with simultaneous reduction in diet for 3 months caused a significant decline in cellulite in $58 \%$ of the patients, whereas in $20 \%$ of the volunteers the effects were only satisfactory [28].

A randomized, double-blind, placebo-controlled trial of the cream Trofolastin ${ }^{\circledR}$, containing an extract of $C$. asiatica, $\alpha$-tocopherol, hydrolyzed collagen and elastin, was carried out on 100 pregnant women. Half of the women, who qualified for the study, received a placebo, the other half were applied a tested cosmetic preparation. The cream was applied daily on breasts, abdomen, buttocks and hips, starting from the third month of pregnancy. The test lasting 30 months was carried through by 80 of the women (39 in the placebo group and 41 in the group using the cream). The results indicated that in the placebo group, striae occurred in $56 \%$ of the women (22 patients), whereas in the group that 
used the cream with the extract of $C$. asiatica, the problem affected 14 women only. The study also assessed the severity of striae on a scale of 0-3. Among the patients using the cream the average numerical value of the parameter adopted was 1.42, and 2.13 among those receiving the placebo. The tested cream provided significant protection for the women who had had striae during puberty (89\% of the women) [29].

\section{Toxicity}

Centella asiatica applied in the recommended doses is not toxic and possible side effects are rare. Centella asiatica may cause: local allergic reactions and burning, especially when used externally and subcutaneously. Skin problems such as eczema on fingers and hands after external use of Madecassol ${ }^{\circledR}$ for a period of 3 months, and a vesicular reaction with pruritus after 20 days of application of Centelase ${ }^{\circledR}$ were observed [30].

\section{Conclusions}

Centella asiatica (Gotu kola) is effective in treatment of wounds, also in infective wounds, burns, and hypertrophic scar. The active compounds include pentacyclic triterpenes, mainly asiaticoside, madecassoside, asiatic and madecassic acids.

The mechanism of action involves promoting fibroblast proliferation and increasing the synthesis of collagen as well as acidic mucopolysaccharides, increasing intracellular fibronectin content and mitotic activity in the germ layer, significantly improving the tensile strength of newly formed skin as well as by inhibiting the inflammatory phase of hypertrophic scars and keloids.

Some studies suggest that the use of $C$. asiatica or its components may be useful in the treatment of psoriasis and scleroderma. Centella asiatica is a common ingredient of cosmetics applied on photoaging skin also in cellulite and striae.

\section{References}

1. Brinkhaus B, Lindner M, Schuppan D, Hahn EG. Chemical, pharmacological and clinical profile of the East Asian medical plant Centella asiatica. Phytomedicine 2000; 75: 427-48.

2. European Pharmacopoeia (Ph. Eur.). Council of Europe, Strasburg 2001.

3. Polish Pharmacopoeia IX. Polish Pharmaceutical Society, Warsaw 2011.

4. Antognoni F, Perellino NC, Crippa S, et al. Irbic acid, a dicaffeoylquinic acid derivative from Centella asiatica cell cultures. Fitoterapia 2011; 8: 2950-4.

5. Gohil KJ, Patel JA, Gajjar AK. Pharmacological review on Centella asiatica: a potential herbal cure-all. Indian J Pharm Sci 2010; 72: 546-56.

6. James J, Dubery I. Identification and quantification of triterpenoid Centelloids in Centella asiatica (L.) urban by densitometric TLC. J Planar Chromatogr 2011; 24: 82-7.

7. Hashin P, Sidek H, Helan MH, et al. Triterpene composition and bioactive of Centella asiatica. Molecules 2011; 16: 1310-22.
8. Centella asiatica. No authors listed. Altern Med Rev 2007; 12: 69-72.

9. European Medicines Agency. Science Medicines Health. http://www.ema.europa.eu, 6.03. 2012.

10. Lu L, Ying K, Wei S, et al. Dermal fibroblast-associated gene induction by asiaticoside shown in vitro by DNA microarray analysis. Br J Dermatol 2004; 151: 571-8.

11. Liu M, Dai Y, Li Y, et al. Madecassoside isolated from Centella asiatica herbs facilitates burn wound healing in mice. Planta Med 2008; 74: 809-15.

12. Lu L, Ying K, Wei S, et al. Asiaticoside induction for cell-cycle progression, proliferation and collagen synthesis in human dermal fibroblasts. Intern J Dermatol 2004; 43: 801-7.

13. Barnes J, Anderson LA, Philipson JD. Herbal medicines. $3^{\text {rd }}$ ed. Pharmaceutical Press, London 2007.

14. WHO Monographs on selected medicinal plants. Vol. 1. World Health Organization, Geneva 1999.

15. Bosse JP, Papillon J, Frenette G, et al. Clinical study of a new antikeloid agent. Ann Plast Surg 1979; 3: 13-21.

16. Widgerow AD, Chait LA, Stals R, Stals PJ. New innovations in scar management. Aesth Plast Surg 2000; 24: 227-34.

17. Maquart FX, Chastang F, Simeon A, et al. Triterpenes from Centella asiatica stimulate extracellular matrix accumulation in rat experimental wounds. Eur J Dermatol 1999; 9: 289-96.

18. Somashekar Shetty BS, Udupa SL, Udupa AL, Somayaji SN. Effect of Centella asiatica L. (Umbelliferae) on normal and dexamethasone suppressed wound healing in Wistar Albino rats. Int J Low Extrem Wounds 2006; 5: 137-43.

19. Shukla A, Rasik AM, Jain GK, et al. In vitro and in vivo wound healing activity of asiaticoside isolated from Centella asiatica. J Ethnopharmacol 1999; 65: 1-11.

20. Shukla A, Rasik AM, Dhawan BN. Asiaticoside-induced elevation of antioxidant levels in healing wounds. Phytother Res 1999; 13: $50-4$.

21. Lee J, Jung E, Kim Y, et al. Asiaticoside induced human collagen I synthesis through TGF-beta Receptor I Kinase (TbetaRI Kinase) - independent smad signaling. Planta Med 2006; 72 : 324-8.

22. Kimura Y, Sumiyoshi M, Samukawa K, et al. Facilitating action of asiaticoside at low doses on burn wound repair and its mechanism. Eur J Pharmacol 2008; 584: 415-23.

23. Sampson JH, Raman A, Karlsen G, et al. In vitro keratinocyte antiproliferant effect of Centella asiatica extract and triterpenoid saponins. Phytomedicine 2001; 8: 230-5.

24. Guseva G, Stravoitova MN, Mach ES. Madecassol treatment of systematic and localized scleroderma. Ter Arkh 1998; 70: 58-61.

25. Haftek M, Mac-Mary S, Le Bitoux MA, et al. Clinical, biometric and structural evaluation of the long-term effects of a topical treatment with ascorbic acid and madecassoside in photoaged human skin. Exp Dermatol 2000; 17: 946-52.

26. Goldman MP, Bacci PA, Leibaschoff G, et al. Cellulite: pathophysiology and treatment. Taylor \& Francis, New York, London 2006.

27. Rossi AB, Vergnanini AL. Cellulite: a review. J Eur Acad Dermatol Venereol 2000; 14: 251-62.

28. Craker LE, Simon JE. Herbs, spices, and medicinal plants: recent advances in botany, horticulture and pharmacology. Vol 3. Food Products Press Binghmanton, New York 1996.

29. Mallol J, Belda MA, Costa D, et al. Prophylaxis of Striae gravidarum with a topical formulation. A double blind trial. Int J Cosmet Sci 1991; 13: 51-7.

30. Gruenwald J, Brendler T, Jaenicke C. PDR for herbal medicines ${ }^{T M}$. $3^{\text {rd }}$ ed. Medical Economics Company Montvale, New Yersey 2004. 